\title{
(C) OPEN ACCESS \\ White matter microstructural alterations in amblyopic adults revealed by diffusion spectrum imaging with systematic tract-based automatic analysis
}

\author{
Tzu-Hsun Tsai, ${ }^{1,2}$ Hsien-Te Su, ${ }^{3}$ Yung-Chin Hsu, ${ }^{3}$ Yao-Chia Shih, ${ }^{4}$ Chien-Chung Chen, ${ }^{5}$ \\ Fung-Rong $\mathrm{Hu}^{1,6}$ Wen-Yih Isaac Tseng ${ }^{3,7}$
}

${ }^{1}$ Department of Ophthalmology, National Taiwan University Hospital, Taipei, Taiwan ${ }^{2}$ Graduate Institute of Clinical Medicine, National Taiwan University College of Medicine, Taipei, Taiwan ${ }^{3}$ Institute of Medical Device and Imaging, National Taiwan University College of Medicine,

Taipei, Taiwan

${ }^{4}$ Institute of Biomedical Engineering, National Taiwan University, Taipei, Taiwan ${ }^{5}$ Department of Psychology, National Taiwan University College of Science, Taipei,

Taiwan

${ }^{6}$ Department of Ophthalmology, National Taiwan University College of Medicine, Taipei, Taiwan

${ }^{7}$ Molecular Imaging Center, National Taiwan University, Taipei, Taiwan

Correspondence to Professor Wen-Yih Isaac Tseng, Institute of Medical Device and Imaging, National Taiwan University College of Medicine, Taipei 10051, Taiwan; wytseng@ntu.edu. tw and Professor FungRong HuDepartment of Ophthalmology, National Taiwan University College of Medicine, Taipei 10051, Taiwan; fungronghu@ntu.edu.tw

F-RH and W-YIT contributed equally.

Received 10 December 2017 Revised 27 February 2018 Accepted 14 May 2018 Published Online First 29 May 2018

Check for updates

To cite: Tsai T-H, Su H-T,

Hsu Y-C, et al.

BrJ Ophthalmol

2019:103:511-516.

\section{ABSTRACT}

Background/aim We investigated the microstructural changes in white matter of adults with amblyopia using diffusion spectrum imaging with systematic tract-based automatic analysis of the whole brain.

Methods Ten adults with amblyopia (six women and four men, $33.6 \pm 10.6$ years old on average) and 20 ageand sex-matched normal-sighted controls were enrolled. The mean generalised fractional anisotropy (GFA) was measured in 76 white matter tracts and compared between the experimental and control groups using a threshold-free cluster-weighted method and $t$-test. A 2-percentile cut-off was used to identify segments with the greatest differences between the two groups. Results Participants with amblyopia had significantly lower GFA values than the controls in 11 segments located in nine white matter tracts, which included the following: left arcuate fasciculus, left frontal aslant tract, left fornix and left inferior fronto-occipital fasciculus of the association fibres; left thalamic radiations of the auditory nerve and bilateral optic radiations of the projection fibres; and genu and middle temporal gyrus of the callosal fibres. Amblyopic participants had statistically higher GFA values in the bilateral uncinate fasciculus than those of the controls.

Conclusion This preliminary study using whole-brain tractographic analysis of white matter reveals association between abnormal early visual processing and alterations in brain architecture, which may be related to various higher-level deficits, such as audiovisual integration and hand-eye coordination in patients with amblyopia.

\section{INTRODUCTION}

Amblyopia, with a prevalence of $2.9 \%-3.2 \%$ in adults, ${ }^{1}$ is a common cause of uncorrectable visual impairment. This neurodevelopmental disorder of the visual cortex occurs when binocular vision is disrupted during early childhood, leads to lifelong visual deficits affecting both monocular and binocular visual function. ${ }^{2}$ Common causes of amblyopia include refraction error, anisometropia, strabismus and visual deprivation arising from ptosis or congenital cataract. ${ }^{12}$

Amblyopia reduces corrected visual acuity and is associated with lower-level visual deficits, for example, reduced contrast sensitivity, and higher-level deficits, for example, those involving global processing tasks (such as form perception, contour processing and motion integration) or abnormal processing of information in the ventral and dorsal pathways. ${ }^{2}$ Abnormal early visual experiences in amblyopia subjects can affect the initial stages of visual-tactile and audiovisual integration and trigger changes in long-term crossmodal plasticity. $^{3-5}$ Additionally, amblyopia also influences motor functions. $^{67}$

Neuroimaging studies have revealed altered anatomical organisation and neural responses in the lateral geniculate nucleus (LGN) and in striate and extrastriate areas of the visual cortex. ${ }^{89}$ Diffusion-weighted magnetic resonance imaging (MRI) is currently one of the main non-invasive techniques used for in vivo mapping of white matter architecture of the human brain. White matter comprises bundles of axons that connect various cortical or subcortical areas. Diffusion tensor imaging (DTI) has been used to study microstructural changes in white matter of amblyopia subjects, leading to the identification of developmental impairments in the optic radiation (OR) and several fasciculi that are associated with dorsal and ventral visual pathways. ${ }^{10-13}$ Diffusion spectrum imaging (DSI) is another kind of diffusion-weighted MRI, which can resolve within-voxel heterogeneity of fibre orientations, analyse crossed fibres within white matter, and follow fibre fascicles into the grey matter of cortical and subcortical regions. ${ }^{14}{ }^{15}$ The advantage of DSI is the ability to generate anatomically complete results and accurately delineate major neural fibres in complex local environments. ${ }^{14}$

When diffusion MRI is combined with tractography, the microstructural properties along a specific fibre tract bundle can be determined manually after delineating regions of interest. However, traditional tract-specific analysis is highly operator-dependent and time-consuming, and thus prone to having reconstruction biases and errors. The newly developed whole-brain tract-based automatic analysis (TBAA) has been used for automated whole-brain investigations of over 76 major white matter tracts, using a high-quality DSI template constructed from images acquired from 122 healthy young adults. This comprehensive and standardised method yields results with high geometrical agreement and considerably less measurement variability than manually reconstructed tracts. ${ }^{16} 17$

We hypothesised that abnormal early visual experience causes alteration of white matter microstructure in regions involved in visual processing and in areas important for linking visual inputs to the correlated motor tasks. Whole-brain investigations 
Table 1 Clinical characteristics of the participants with amblyopia

\begin{tabular}{|c|c|c|c|c|c|c|c|c|c|c|}
\hline \multirow[b]{2}{*}{ No. } & \multirow[b]{2}{*}{ Sex } & \multirow{2}{*}{$\begin{array}{l}\text { Age, } \\
\text { years }\end{array}$} & \multirow[b]{2}{*}{$\mathrm{AE}$} & \multirow[b]{2}{*}{ Handedness } & \multirow{2}{*}{$\begin{array}{l}\text { BCVA } \\
R\end{array}$} & \multirow{2}{*}{$\begin{array}{l}\text { BCVA } \\
\text { L }\end{array}$} & \multirow{2}{*}{$\begin{array}{l}\text { Refraction } \\
R\end{array}$} & \multirow{2}{*}{$\begin{array}{l}\text { Refraction } \\
\mathrm{L}\end{array}$} & \multirow{2}{*}{ Education } & \multirow{2}{*}{ Others } \\
\hline & & & & & & & & & & \\
\hline 1 & M & 48 & $\mathrm{R}$ & L & 0.1 & 1.5 & $-2.50 /-2.00,15^{\circ}$ & $-1.75 /-0.75,150^{\circ}$ & middle school & RXT, cataract \\
\hline 2 & M & 35 & L & $\mathrm{R}$ & 1.5 & 0.1 & $-4.50 /-0.75,170^{\circ}$ & $+5.0 /-1.25,180^{\circ}$ & graduate school & \\
\hline 3 & M & 23 & $\mathrm{R}$ & $\mathrm{R}$ & 0.6 & 1.0 & $-1.50 /-4.25,10^{\circ}$ & Plano & college & RXT \\
\hline 4 & $\mathrm{~F}$ & 28 & $\mathrm{R}$ & $\mathrm{R}$ & 0.6 & 1.2 & +4.75 & -0.25 & college & \\
\hline 5 & $\mathrm{~F}$ & 29 & $R$ & $\mathrm{R}$ & 0.3 & 1.0 & $+2.25 /-0.75,162^{\circ}$ & $-4.25 /-0.50,180^{\circ}$ & college & \\
\hline 6 & $\mathrm{~F}$ & 30 & L & $\mathrm{R}$ & 1.0 & 0.6 & $-3.75 /-0.75,175^{\circ}$ & $+0.25 /-1.75,180^{\circ}$ & high school & \\
\hline 7 & $\mathrm{~F}$ & 49 & L & L & 1.0 & 0.1 & $-6.0 /-0.75,180^{\circ}$ & $-1.25 /-0.75,180^{\circ}$ & high school & \\
\hline 8 & $\mathrm{~F}$ & 24 & $\mathrm{R}$ & $\mathrm{R}$ & 0.1 & 0.5 & $-3.5 /-0.50,160^{\circ}$ & $0 /-2.00,15^{\circ}$ & college & \\
\hline 9 & $\mathrm{~F}$ & 47 & L & L & 1.2 & 0.15 & -0.25 & $+3.5 /-0.25,130^{\circ}$ & graduate school & \\
\hline 10 & M & 23 & $\mathrm{R}$ & $\mathrm{R}$ & 0.05 & 1.0 & $-8.00 /-1.00,15^{\circ}$ & $-6.75 /-1.25,160^{\circ}$ & college & RXT \\
\hline
\end{tabular}

$A E$, affected eye; $B C V A$, best-corrected visual acuity; RXT, right eye exotropia.

with DSI and TBAA were performed in this study to systematically compare white matter integrity in amblyopic adults with that in normal-sighted controls.

\section{METHODS}

\section{Participants}

The experimental group consisted of subjects aged 20-50 years diagnosed with amblyopia in the ophthalmology clinic at the National Taiwan University Hospital. Amblyopia was defined with a best-corrected visual acuity of less than 20/20 in one or both eyes or a difference of best-corrected visual acuity more than two lines between two eyes. Anisometropic, strabismic, as well as deprivated amblyopia were included in the study. Subjects with neurological or psychiatric disease were excluded. A full-time paediatric ophthalmologist (T-HT), assessed and diagnosed all the subjects. For every amblyopic subject, two age- and sex-matched subjects without strabismus or amblyopia and with normal stereovision were recruited by poster in local communities around the National Taiwan University as controls. All participants underwent a best-corrected visual acuity test, slit lamp and fundus examination, stereotest with random-dot stereogram 300" (NTU 300") and ocular motility test.

The mean age $\pm S D$ was $33.6 \pm 10.6$ years in the amblyopia group and $33.6 \pm 10.2$ years in the control group $(p=0.88)$. There were six women in the amblyopia group (6/10) and 12 in the control group (12/20). Table 1 summarises the demographic data of the amblyopia group. Subject 1 had history of monocular congenital cataract and underwent surgery at 4 years of age. Subjects 1, 3 and 10 had exotropia in the affected eye. None of the amblyopic participants passed the screening with random-dot stereogram 300".

This study complied with the Health Insurance Portability and Accountability Act. Written informed consent was obtained prospectively from the study participants after detailed explanations, according to a protocol conforming to the tenets of the Declaration of Helsinki.

\section{MRI acquisition}

MR images were acquired using a 3 T MRI system (Trio, Siemens, Erlangen, Germany) with a 32-channel phased-array head coil. The slice orientation of the axial images was defined on the sagittal localiser as the orientation parallel to the line between the anterior and posterior commissures. High-resolution T1-weighted images were acquired using a three-dimensional magnetisation-prepared rapid gradient-echo sequence (parameters: repetition time $=2000 \mathrm{~ms}$; echo time $=3 \mathrm{~ms}$; flip angle $=9^{\circ}$; matrix size $=256 \times 192 \times 208$; field of view $=256 \times 192 \times 208$ $\mathrm{mm}^{3}$ ), resulting in an isotropic spatial resolution of $1 \mathrm{~mm}^{3}$. DSI was performed using a single-shot spin-echo echo-planar imaging sequence with twice-refocused balanced echo for reducing eddy current distortions. Thirty-nine axial images that covered the entire cerebrum were acquired contiguously (parameters: repetition time $=9600 \mathrm{~ms}$; echo time $=130 \mathrm{~ms}$; matrix size $=80 \times 80$; field of view $=200 \times 200 \mathrm{~mm}^{2}$; slice thickness $=2.5$ $\mathrm{mm})$. According to a modified optimum sampling scheme, a total of 102 volumes of diffusion-weighted images was acquired with diffusion-encoding gradient directions pointing at the grid points within a half-sphere of the diffusion-encoding space (q-space); the maximum diffusion sensitivity (bmax) was 4000 $\mathrm{s} / \mathrm{mm}^{2}$.

\section{DSI data reconstruction and TBAA}

Before reconstruction, the DSI data underwent quality assurance entailing signal drop-out screening, signal-to-noise ratio screening and shaking artefact correction. For each voxel, the 102 diffusion samples were processed using ReMAP estimation, which is a regularised version of Mean Apparent Propagator MRI. ${ }^{18}$ The generalised fractional anisotropy (GFA) was calculated from the orientation distribution function. GFA represents the diffusion-relevant anisotropy of local architectures, which infers the microstructural integrity of white matter, analogous to the use of fractional anisotropy (FA) in DTI. It ranges from 0 (completely isotropic diffusion) to 1 (diffusion is markedly restricted to one dimension). ${ }^{19}$ The technical details of TBAA have been described previously. ${ }^{17} 17$ The position coordinates of the 76 tracts were transformed from the NTU-DSI122 template to the individual DSI data sets with the deformation maps, and the coordinates were aligned with the proceeding direction of each tract bundle. Each aligned tract was normalised and divided into 100 sampling steps along the sampling coordinates and recorded as the sampling steps in the template space. Linear regression analysis was used to remove the effects of age and sex in each step. The GFA values were sampled in the native space along the sampling steps. Finally, a 3D connectogram (x-axis: steps along sampling coordinates; $y$-axis: 76 white matter tracts; $z$-axis: number of each subject) was obtained as the output of TBAA.

\section{Threshold-free Cluster-weighted (TFCW) Scores for group analysis}

To compare the differences between the two groups by looking at each sampling step of the tracts and finding the steps with the most significant differences, a total of $76 \times 100$ comparisons had to be made. Since no significance of difference would be retained after correcting for this number of multiple 

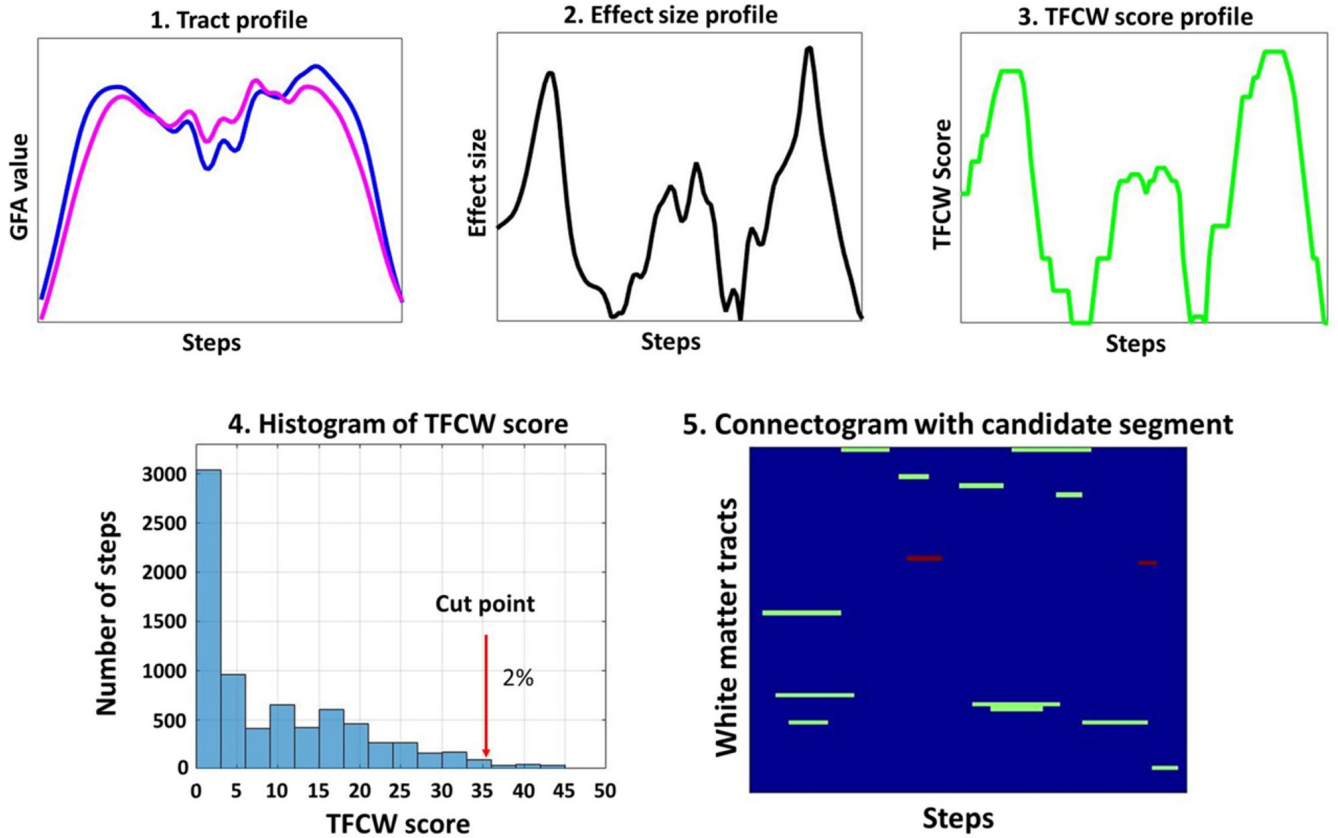

Figure 1 Threshold-free Cluster-weighted (TFCW) analysis. (1) Tract profiles of the callosal fibres of genu in average generalised fractional anisotropy (GFA) are shown as representative tracts (normal-sighted controls: blue; subjects with amblyopia: magenta). (2) Computing the effect size for each step. (3) Calculating the TFCW Score for each step. (4) Steps with scores in the top 2-percentiles of the histogram were selected. (5) The green segments indicate higher GFA values in controls than in subjects with amblyopia. The red segments indicate lower GFA values in controls than in subjects with amblyopia.

comparisons, we adopted a statistical method proposed by Smith and Nichols using TFCW Scores. ${ }^{20}$ The TFCW Score rescaled the effect size (ES) at each step by considering the contributions of the lengths of continuous segments at different thresholds.

The TFCW Score $(S p)$ for step $p$ was defined as:

$$
S_{p}=\sum_{e=0.1}^{1} C_{p}(e) \cdot E S_{p}
$$

Threshold levels (e) for ES were set from 0.1 to 1 in 10 aliquots, while the $C_{p}(e)$ was the segment step size at the threshold level e. The value of $S_{p}$ was simply $E S$ at step $p\left(E S_{p}\right)$ multiplied by an area extended from step $p$ under the ES profile (figure 1). This area was equivalent to the length summation of the continuous step segments that contained step $p$. After calculating the $S_{p}$ of all the steps in the connectogram, we plotted a histogram with TFCW Scores (7600 in total) and selected those in the top 2-percentile as the segments with noticeable between-group differences. Figure 1 shows the procedures in the analysis with TFCW Scores. Although the validity of the results seems less strong than that obtained from standard statistics with multiple comparison correction, it is better than those simply using uncorrected $\mathrm{p}$ values with a certain threshold of the cluster size. Mean GFA values of the segments that passed the 2-percentile cut-off were calculated and compared by $t$-test between two groups for further validation.

\section{RESULTS}

Data from 10 amblyopia subjects and 20 matched controls were analysed. We identified 162 steps that were in the top 2-percentile of the histogram. These steps formed 14 segments in 12 tracts (figures 2-4). Among the association fibres, the tracts included the left arcuate fasciculus (AF), left frontal aslant tract (FAT), left fornix, left inferior fronto-occipital fasciculus (IFOF), and bilateral uncinate fasciculi (figures 2 and 3). Among the callosal fibres
(CF), these tracts were the CF of the genu and the CF connecting the middle temporal gyri (figure 3). Among the projection fibres, the tracts were the left frontal striatum (FS) of the orbitofrontal cortex (OFC), left thalamic radiations (TRs) of the auditory nerve and the optic radiations in both hemispheres (figure 4).

The mean GFA values of these 12 white matter tracts are listed in table 2. The left AF and CF of the genu contained two segments each; therefore, the mean GFA values were determined by averaging the GFA values over the two segments on each tract. In 11 of the 12 tracts, the GFA values were significantly different between the amblyopia and the control groups $(\mathrm{p}<0.05)$. Subjects with amblyopia had higher mean GFA values in bilateral uncinate fasciculi and lower mean GFA values in the other nine tracts compared with controls. The GFA value in the segment of the left FS of the OFC was slightly lower in the amblyopia group than in the control, although not statistically significantly $(\mathrm{p}=0.072)$.

\section{DISCUSSION}

Using DSI with TBAA to assess subjects with amblyopia, we found 12 white matter tracts containing segments with differences that were ranked in the top 2-percentile as compared with healthy controls. Results of the whole-brain tractography suggested association between abnormal early visual processing and brain architectural changes.

Early visual experience is essential in building the neural network of the visual system. Although white matter architecture can change with age and learning experience, neural plasticity is at its highest in young children during a period termed 'the critical period of vision development'. Disruption of visual input during this period may result in amblyopia, and functional recovery is difficult when neuroplasticity slows down at the end of the critical period. Amblyopia had been found to be associated with changes of functional brain organisation in primary 

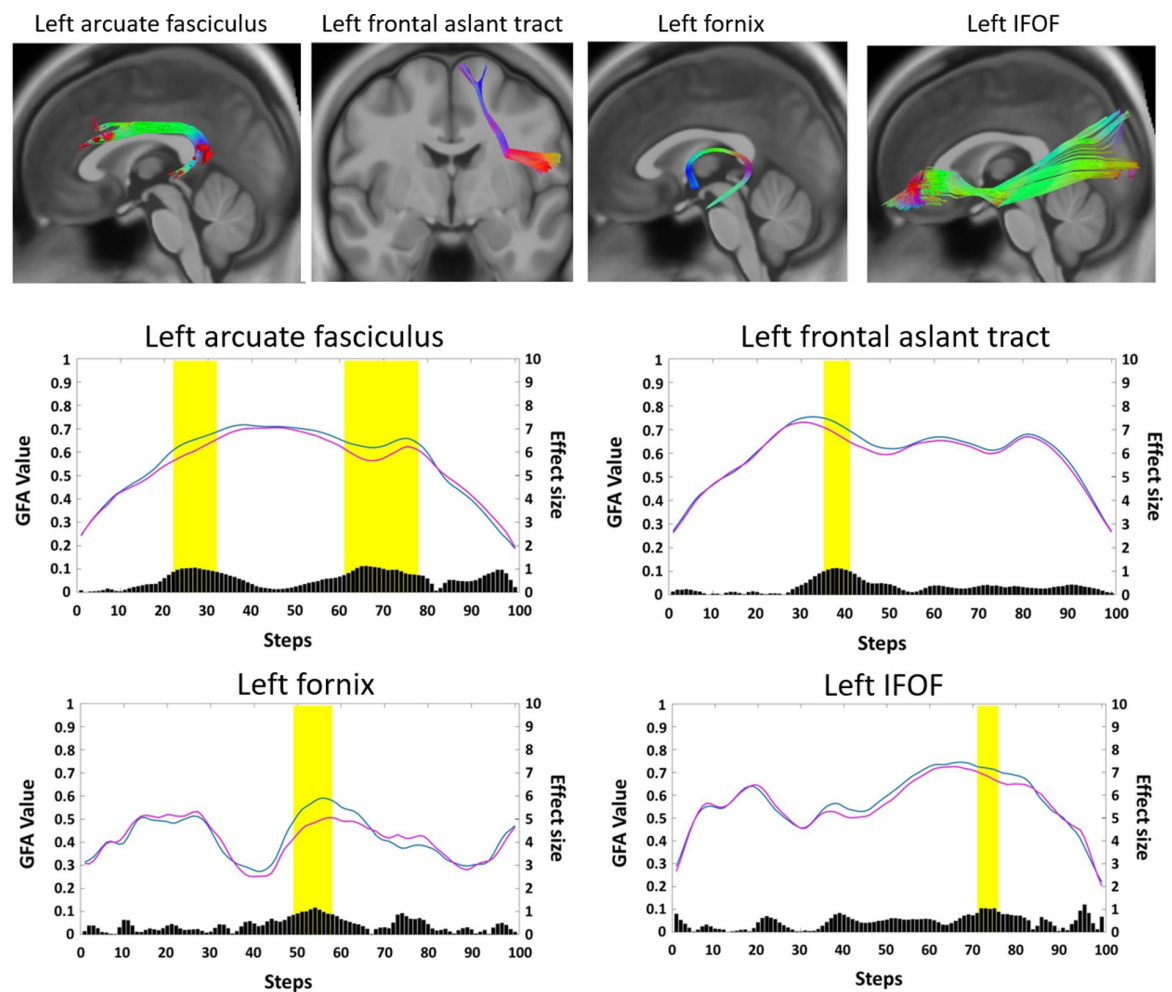

Figure 2 The threshold-free cluster-weighted (TFCW) method identified four association fibre tracts that contained segments that were different between the two study groups. Normal-sighted controls, blue; subjects with amblyopia, magenta; black bars under the tract profile indicate the effect size for each step; yellow rectangles indicate the segments that stand out as top 2-percentile differences. GFA, generalised fractional anisotropy; IFOF, inferior fronto-occipital fasciculus.
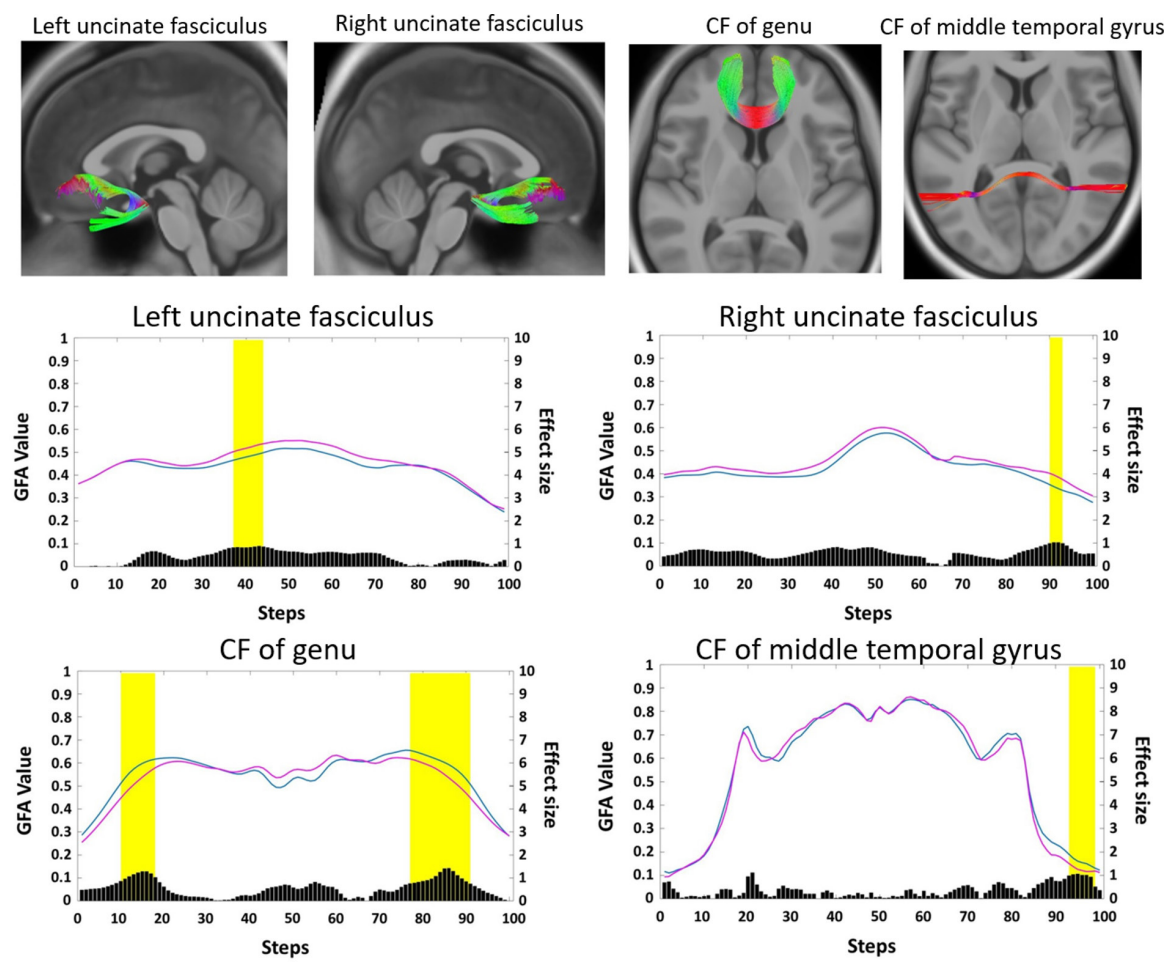

Figure 3 The threshold-free cluster-weighted (TFCW) method identified two association fibre tracts and two callosal fibre (CF) tracts that contained segments that were different between the two study groups. Normal-sighted controls, blue; subjects with amblyopia, magenta; black bars under the tract profile indicate the effect size for each step; yellow rectangles indicate the segments that stand out as top 2-percentile differences. GFA, generalised fractional anisotropy. 

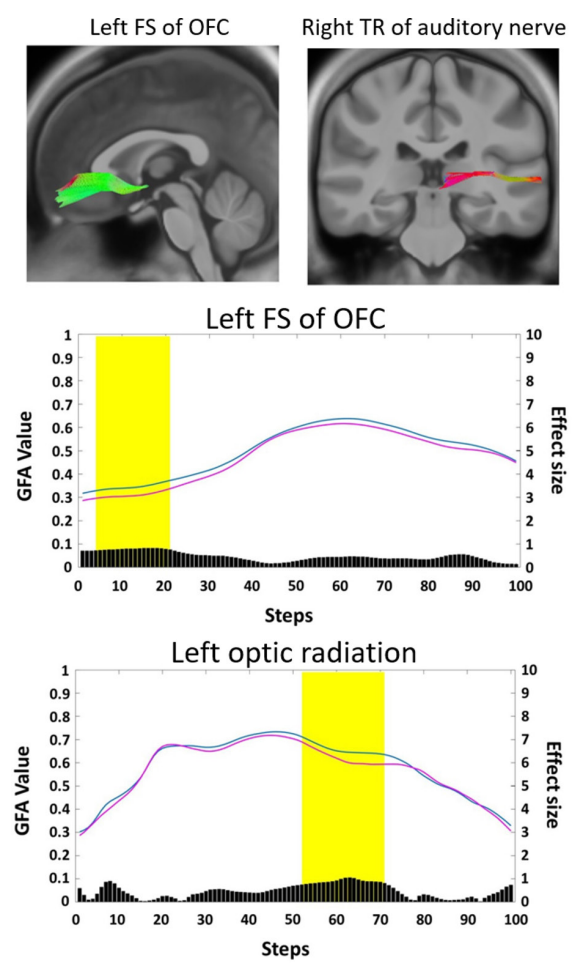
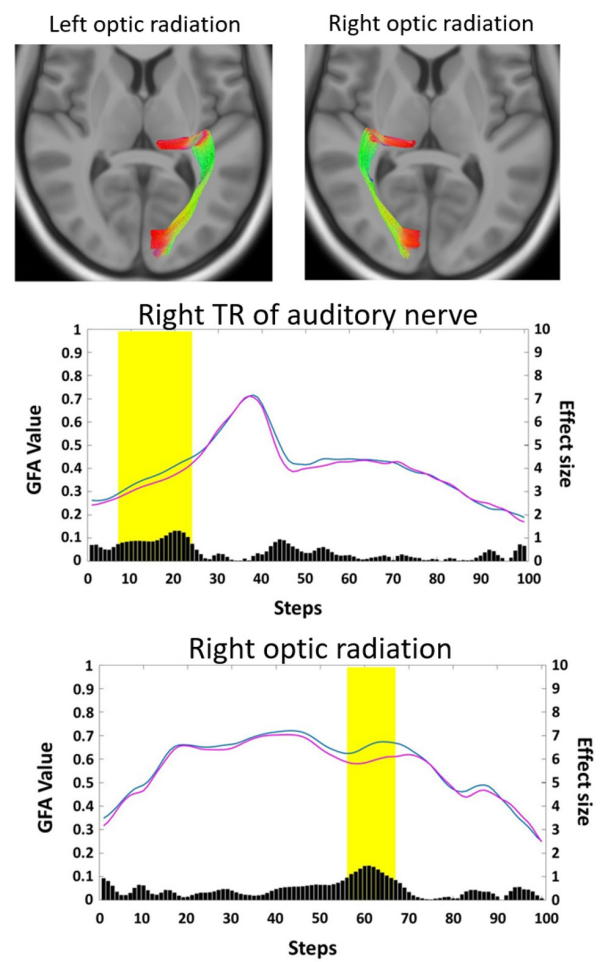

Figure 4 The threshold-free cluster-weighted (TFCW) method identified four projection fibre tracts that contained segments that were different between the two study groups. Normal-sighted controls, blue; subjects with amblyopia, magenta; black bars under the tract profile indicate the effect size for each step; yellow rectangles indicate the segments that stand out as top 2-percentile differences. FS, frontal striatum; GFA, generalised fractional anisotropy; OFC, orbitofrontal cortex; TR, thalamic radiation.

visual cortex and LGN, volume loss in visual cortical regions and alteration of white matter fasciculi associated with visual processing. ${ }^{8-13}$

On comprehensive evaluation of major white matter fibre architecture in amblyopic adults, we found that the amblyopia group had lower mean GFA values in fascicles involved in visual processing. The OR connects the visual cortex and the LGN, and the IFOF is associated with the ventral stream of visual

Table 2 The threshold-free cluster-weighted (TFCW) method revealed 12 white matter tracts with different generalised fractional anisotropy (GFA) values between amblyopic adults and normalsighted controls

\begin{tabular}{lllll}
\hline \multirow{2}{*}{$\begin{array}{l}\text { Tract } \\
\text { No. }\end{array}$} & Name of tracts & \multicolumn{2}{l}{$\begin{array}{l}\text { GFA values } \\
\text { Mean } \pm \text { SD }\end{array}$} & \\
\cline { 2 - 4 } & & Controls & Amblyopia & \\
\hline 1 & Left optic radiation & $0.659 \pm 0.038$ & $0.623 \pm 0.038$ & 0.0176 \\
\hline 2 & Right optic radiation & $0.654 \pm 0.055$ & $0.594 \pm 0.044$ & 0.0038 \\
\hline 3 & Left IFOF & $0.717 \pm 0.026$ & $0.682 \pm 0.047$ & 0.0072 \\
\hline 4 & Left arcuate fasciculus & $0.643+0.026$ & $0.598 \pm 0.044$ & 0.0022 \\
\hline 5 & Left TR of the auditory nerve & $0.371 \pm 0.030$ & $0.342 \pm 0.030$ & 0.0110 \\
\hline & CF of the middle temporal & & & \\
6 & gyrus & $0.161 \pm 0.041$ & $0.128 \pm 0.018$ & 0.0111 \\
7 & Left frontal aslant tract & $0.609 \pm 0.054$ & $0.554 \pm 0.043$ & 0.0063 \\
\hline 8 & Left fornix & $0.560 \pm 0.079$ & $0.475 \pm 0.088$ & 0.0102 \\
9 & CF of the genu & $0.592 \pm 0.037$ & $0.539 \pm 0.042$ & 0.0007 \\
\hline 10 & Left uncinate fasciculus & $0.482 \pm 0.050$ & $0.520 \pm 0.045$ & 0.0022 \\
11 & Right uncinate fasciculus & $0.341 \pm 0.033$ & $0.390 \pm 0.068$ & 0.0056 \\
12 & Left FS of the OFC & $0.346 \pm 0.048$ & $0.311 \pm 0.046$ & 0.0720 \\
\hline
\end{tabular}

$\mathrm{CF}$, callosal fibres; $\mathrm{FS}$, frontal striatum; IFOF, inferior fronto-occipital fasciculus; OFC, orbitofrontal cortex; TR, thalamic radiation pathway. A previous study that used DTI to investigate children with anisometropic amblyopia showed a bilateral decrease in the FA in the OR. ${ }^{10}{ }^{12}$ In another DTI study, altered FA values were found in the right OR, left inferior longitudinal fasciculus and IFOF. $^{11}$

Interestingly, recent studies showed that amblyopia is associated with altered 'audiovisual integration,' which manifests as a reduced McGurk effect. This defect of fusing incongruent auditory and visual syllables may persist into adulthood in patients with amblyopia. ${ }^{45}$ Some of the altered white matter tracts found in our study are known to be involved in language and auditory functions. The AF is one of the four subcomponents of the superior longitudinal fasciculus, and is regarded as a part of the dorsal stream for language which plays a role in mapping acoustic sounds to articulatory representations. ${ }^{21}$ The left TR of the auditory nerve connects the thalamus to Heschl's gyrus, which processes incoming auditory information. The CF connecting the middle temporal gyri may be involved in language processing. ${ }^{22}$

Some authors reported that children with amblyopia were impaired in fine motor skill and hand-eye coordination tasks, particularly manual dexterity tasks requiring speed and accuracy. ${ }^{67}$ The frontal aslant tract (FAT), which connects the inferior frontal gyrus with the supplementary and presupplementary motor areas, is regarded as part of a 'motor stream' involved in visuomotor processing. The bilateral FAT is associated with the closing grip phase of hand kinematics, which determines efficient and stable grasping of a target object. ${ }^{23}$ Altered FAT might be involved in the neuromechanism underlying defective upper limb coordination in patients with amblyopia.

In a resting-state functional MRI study, Lin et al found that adults with anisometropic amblyopia had reduced regional homogeneity in the prefrontal cortices and the OFC. ${ }^{24}$ 
Interestingly, our study found that the GFA value was lower for a segment of CFs of the genu, which connect the bilateral prefrontal cortices and OFCs. The uncinate fasciculi are bidirectional, long-range white matter tract that connects lateral OFCs to the anterior temporal lobes, and they were the only white matter tracts of the amblyopia group with higher mean GFA values than those of the controls in the present study. We speculated that it is the result of overcompensation during development as the uncinate fasciculus transmits information from the visual ventral stream to the OFC for visual discrimination.

To our knowledge, DSI and TBAA have not previously been used to investigate white matter organisation in adults with amblyopia. Compared with previous studies of human amblyopia using white matter tractography, the major advantage of our study is that we were able to screen 76 major fibre tracts comprehensively. However, without assessing behavioural parameters and structural MRI data in the same patients, any attempt to link the present data to behaviour must remain speculative.

The primary limitation of our study is its small sample size. A larger study with participants from different age groups and categorisation of amblyopia is warranted, and the correlation between the effect of eye dominance, visual acuity and the level of stereopsis on white matter structure should be analysed to gain a better understanding of neural mechanisms underlying amblyopia.

In conclusion, in this preliminary study, we found reduced GFA in 11 segments of nine white matter fibre tracts in adults with amblyopia. Our results suggest that abnormal early visual experience may induce structural alterations in white matter of the brain, which may be associated with various deficits, such as visual processing, audiovisual integration and hand-eye coordination. TBAA and DSI are potentially promising tools for evaluating microstructural integrity in individuals with amblyopia and for elucidating the possible neural mechanisms underlying the disease symptoms.

Acknowledgements The authors thank the Department of Medical Research at National Taiwan University Hospital, for providing the English editing.

Contributors Substantial contributions to the conception or design of the work, or the acquisition, analysis or interpretation of data: T-HT; H-TS; Y-CH; Y-CS; C-CC; W-YIT. Drafting the work or revising it critically for important intellectual content: T-HT; H-TS; F-RH; W-YIT. Final approval of the version published: W-YIT and F-RH.

Funding This work was supported by the Ministry of Science and Technology, R.O.C. grant number NSC 101-2410-H-002-095 and NSC 102-2420-H-002-016MY2.

Competing interests None declared.

Patient consent Parental/guardian consent obtained.

Ethics approval The Institutional Review Board of National Taiwan University Hospital, Taipei, Taiwan.

Provenance and peer review Not commissioned; externally peer reviewed.

Open access This is an open access article distributed in accordance with the Creative Commons Attribution Non Commercial (CC BY-NC 4.0) license, which permits others to distribute, remix, adapt, build upon this work non-commercially, and license their derivative works on different terms, provided the original work is properly cited, appropriate credit is given, any changes made indicated, and the use is non-commercial. See: http://creativecommons.org/licenses/by-nc/4.0

(C) Author(s) (or their employer(s)) 2019. Re-use permitted under CC BY-NC. No commercial re-use. See rights and permissions. Published by BMJ.

\section{REFERENCES}

1 Attebo K, Mitchell P, Cumming R, et al. Prevalence and causes of amblyopia in an adult population. Ophthalmology 1998;105:154-9.

2 Wong AM. New concepts concerning the neural mechanisms of amblyopia and their clinical implications. Can J Ophthalmol 2012;47:399-409.

3 Niechwiej-Szwedo E, Chin J, Wolfe PJ, et al. Abnormal visual experience during development alters the early stages of visual-tactile integration. Behav Brain Res 2016;304:111-9.

4 Burgmeier R, Desai RU, Farner KC, et al. The effect of amblyopia on visual-auditory speech perception: why mothers may say "Look at me when I'm talking to you". JAMA Ophthalmol 2015;133:11-16.

5 Narinesingh C, Goltz HC, Raashid RA, et al. Developmental trajectory of McGurk effect susceptibility in children and adults with amblyopia. Invest Ophthalmo/ Vis $\mathrm{SC}$ 2015;56:2107-13.

6 Webber AL, Wood JM, Gole GA, et al. The effect of amblyopia on fine motor skills in children. Invest Ophthalmol Vis Sci 2008;49:594-603.

7 Suttle CM, Melmoth DR, Finlay AL, et al. Eye-hand coordination skills in children with and without amblyopia. Invest Ophthalmol Vis Sci 2011;52:1851-64.

8 Hess RF, Thompson B, Gole G, et al. Deficient responses from the lateral geniculate nucleus in humans with amblyopia. Eur J Neurosci 2009;29:1064-70.

9 Mendola JD, Conner IP, Roy A, et al. Voxel-based analysis of MRI detects abnormal visual cortex in children and adults with amblyopia. Hum Brain Mapp 2005;25:222-36.

10 Li Q, Jiang Q, Guo M, et al. Grey and white matter changes in children with monocular amblyopia: voxel-based morphometry and diffusion tensor imaging study. Br J Ophthalmol 2013;97:524-9.

11 Li Q, Zhai L, Jiang Q, et al. Tract-based spatial statistics analysis of white matter changes in children with anisometropic amblyopia. Neurosci Lett 2015;597:7-12.

12 Qi S, Mu YF, Cui LB, et al. Association of optic radiation integrity with cortical thickness in children with anisometropic amblyopia. Neurosci Bull 2016;32:51-60.

13 Duan Y, Norcia AM, Yeatman JD, et al. The structural properties of major white matter tracts in strabismic amblyopia. Invest Ophthalmol Vis Sci 2015;56:5152-60.

14 Wedeen VJ, Wang RP, Schmahmann JD, et al. Diffusion spectrum magnetic resonance imaging (DSI) tractography of crossing fibers. Neuroimage 2008;41:1267-77.

15 Wedeen VJ, Hagmann P, Tseng WY, et al. Mapping complex tissue architecture with diffusion spectrum magnetic resonance imaging. Magn Reson Med 2005;54:1377-86.

16 Hsu YC, Lo YC, Chen YJ, et al. NTU-DSI-122: A diffusion spectrum imaging template with high anatomical matching to the ICBM-152 space. Hum Brain Mapp 2015;36:3528-41.

17 Chen YJ, Lo YC, Hsu YC, et al. Automatic whole brain tract-based analysis using predefined tracts in a diffusion spectrum imaging template and an accurate registration strategy. Hum Brain Mapp 2015:36:3441-58.

18 Özarslan E, Koay CG, Shepherd TM, et al. Mean apparent propagator (MAP) MRI: a novel diffusion imaging method for mapping tissue microstructure. Neuroimage 2013;78:16-32.

19 Yeh FC, Wedeen VJ, Tseng WY. Estimation of fiber orientation and spin density distribution by diffusion deconvolution. Neuroimage 2011;55:1054-62.

20 Smith SM, Nichols TE. Threshold-free cluster enhancement: addressing problems of smoothing, threshold dependence and localisation in cluster inference. Neuroimage 2009;44:83-98.

21 Saur D, Kreher BW, Schnell S, et al. Ventral and dorsal pathways for language. Proc Natl Acad Sci U S A 2008;105:18035-40.

22 Cabeza R, Nyberg L. Imaging cognition II: An empirical review of 275 PET and fMRI studies. J Cogn Neurosci 2000;12:1-47.

23 Budisavljevic S, Dell'Acqua F, Djordjilovic V, et al. The role of the frontal aslant tract and premotor connections in visually guided hand movements. Neuroimage 2017;146:419-28.

24 Lin X, Ding K, Liu Y, et al. Altered spontaneous activity in anisometropic amblyopia subjects: revealed by resting-state FMRI. PLoS One 2012;7:e43373. 